\title{
Strong Influence of Hole Shape on Extraordinary Transmission through Periodic Arrays of Subwavelength Holes
}

\author{
K. J. Klein Koerkamp, ${ }^{1}$ S. Enoch,${ }^{2}$ F. B. Segerink, ${ }^{1}$ N. F. van Hulst, ${ }^{1}$ and L. Kuipers ${ }^{1,3}$ \\ ${ }^{1}$ Applied Optics Group, MESA+ Institute for Nanotechnology and Department of Science \& Technology, University of Twente, \\ P.O. Box 217, 7500 AE Enschede, The Netherlands \\ ${ }^{2}$ Institut Fresnel, CNRS 6133, Campus de St. Jérôme, case 161, 13397 Marseille Cedex 20, France \\ ${ }^{3}$ FOM-Institute for Atomic and Molecular Physics (AMOLF), Kruislaan 407, 1098 SJ Amsterdam, The Netherlands \\ (Received 7 March 2003; revised manuscript received 4 November 2003; published 4 May 2004)
}

\begin{abstract}
We show that extraordinary light transmission of periodic subwavelength hole arrays, generally attributed to surface-plasmon resonances, is strongly influenced by the hole shape. Both experiments and calculations, based on a Fourier modal method, demonstrate that a shape change from circular to rectangular increases the normalized transmission by an order of magnitude while the hole area decreases. Moreover, the spectra exhibit large redshifts $\left(\sim 2500 \mathrm{~cm}^{-1}\right)$. A comparison with the transmission of isolated holes shows that shape resonances of the rectangular holes play a dominant role.
\end{abstract}

PACS numbers: 42.79.Dj, 71.36.+c, 73.20.Mf, 78.66.Bz

For the manipulation of light at a subwavelength scale with artificial photonic structures two ingredients exist: shape and periodicity. On the one hand, the shape of subwavelength structures is used, for example, to create high local fields [1], shift the spectrum of localized resonances [2], or induce new and tailor-made responses [3]. As shape strongly affects the response of metal particles $[2,4]$, shape may also be expected to affect the transmission through holes, as these shapes and holes are, to a large extent, complementary geometries. The periodicity of the structures, on the other hand, is used to produce band gaps in the dispersion relation of photons [5] and plasmons [6], which yield a huge control over the photons and plasmons. A beautiful example of light manipulation within periodic arrangements of subwavelength air holes in thick metal films was the generation of so-called extraordinary transmission: for certain colors the transmitted fraction of the incident light exceeds the open fraction of the film [7]. The enhanced transmission has been attributed to a resonant excitation of surface-plasmon polaritons set up by the periodicity $[8,9]$. Recently, similar arrays were used to transfer quantum entanglement from photons to plasmons [10]. The theoretical description of the extraordinary transmission is an active research field [11-14]. For photonic crystals it has been theoretically predicted that combining shape and periodicity should lead to enhanced optical properties, such as an increased width of the photonic band gap $[15,16]$.

In this Letter, we show, experimentally and theoretically, that combining shape and periodicity profoundly affects the extraordinary transmission phenomenon. By changing the shape of the holes, the normalized transmission cannot only be enhanced by an order of magnitude even though the hole size actually decreases, but the central wavelength of the transmitted peak intensity can also be substantially influenced. Theoretical calculations based on a Fourier modal method confirm the experimental findings. From the optical properties of isolated holes, we conclude that shape resonances, which are in effect localized modes, within the individual rectangular holes, dominate the properties of the periodic arrays.

The periodic air hole arrays were fabricated by ion milling in optically thick $(200 \mathrm{~nm})$ Au films deposited on glass with a square lattice and an equal period of $d=$ $425 \mathrm{~nm}$. Figure 1 presents focused ion beam images of two of the investigated arrays. Figure 1(a) shows an array consisting of circular holes with a diameter of $190 \mathrm{~nm}$; Fig. 1(b) depicts an array with rectangular holes $(75 \times$ $225 \mathrm{~nm}^{2}$ ). Figure 1 shows the uniformity of the hole shapes and the edge sharpness of the fabricated structures (better than $10 \mathrm{~nm}$ ). The third investigated array (not shown here) consists of rectangular air holes of $150 \times$ $225 \mathrm{~nm}^{2}$.

The transmission through the air hole arrays is measured with a linearly polarized broadband light source in combination with an optical multichannel analyzer. The range of wavelengths investigated with the setup is
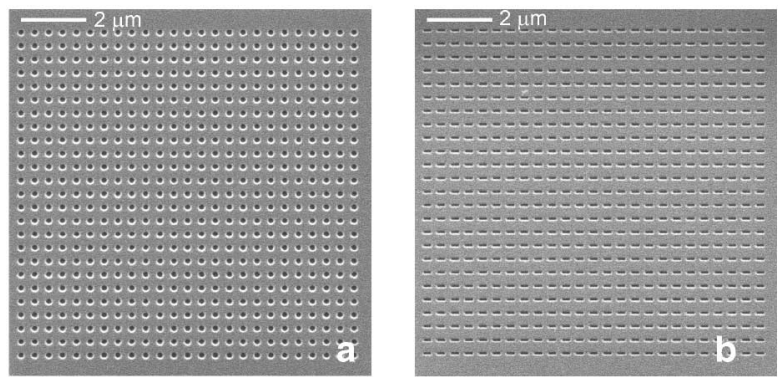

FIG. 1. Focused ion beam images of two periodic subwavelength hole arrays fabricated in a $200 \mathrm{~nm}$ thick gold film. Both arrays have a period of $425 \mathrm{~nm}$. (a) An array consisting of circular holes with a diameter of $190 \mathrm{~nm}$. (b) An array consisting of rectangular holes of $75 \times 225 \mathrm{~nm}^{2}$. 
$480-1000 \mathrm{~nm}$ with a resolution of $3 \mathrm{~nm}$. The incoming beam is slightly focused (numerical aperture $=0.03$ ) and the 0th order transmission is detected. The transmission of the arrays is measured and normalized to the open fraction of the array in order to obtain the transmissivity of the holes. This quantity is introduced to allow a direct comparison of arrays with differently sized and shaped holes. Figure 2 presents the measured transmissivity through the three different air hole arrays, showing that the shape of the holes has a tremendous effect on the transmissivity. The array with the circular air holes exhibits the maxima and minima of the extraordinary transmission also found by others $[8,10]$. In Fig. 2 the $(1,0),(1,1)$, and $(2,0)$ order maxima for the circular hole array are visible along with the corresponding minima at slightly shorter wavelengths. All the minima are associated with so-called Wood's anomalies [17]. The maximum transmissivity for the peaks in the Au films is less than that observed for $\mathrm{Ag}$ films [7]. This difference is probably associated with a stronger damping of surface plasmons on Au with respect to Ag in this wavelength range [18].

The transmissivity changes dramatically when the shape of the holes is changed. For the array consisting of $150 \times 225 \mathrm{~nm}^{2}$ rectangles the $(1,0)$ maximum is 1.9 , i.e., an increase by a factor of 7.3 with respect to the array with the circular holes. This large enhancement cannot be simply explained by the $19 \%$ increase in hole area of the $150 \times 225 \mathrm{~nm}^{2}$ rectangles with respect to the $190 \mathrm{~nm}$ diameter circular holes. When the size of the rectangles

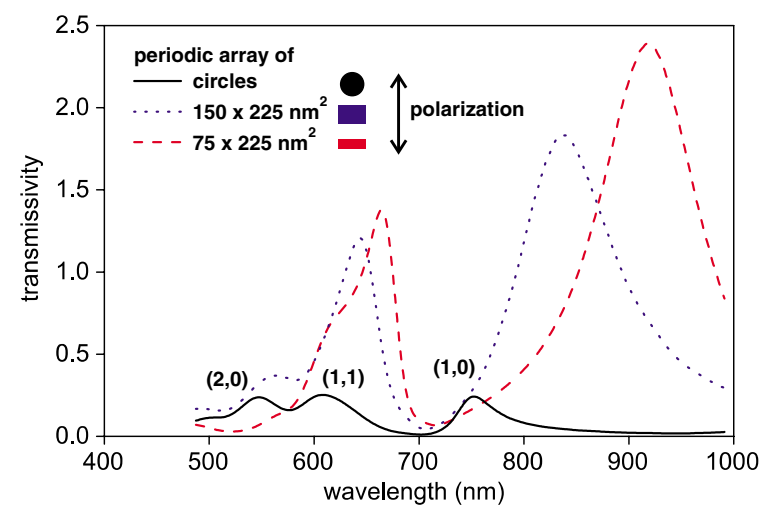

FIG. 2 (color online). Measured transmissivity spectra for three different subwavelength hole arrays. The spectra are measured for arrays consisting of circular holes (solid line), $150 \times 225 \mathrm{~nm}^{2}$ rectangles (dotted line), and $75 \times 225 \mathrm{~nm}^{2}$ rectangles (dashed line). The numbers inside parentheses specify the order of the maxima. The polarization of the incoming light was perpendicular to the long axis of the rectangles. Both the height of the transmissivity and the position of the transmissivity peaks are greatly affected by the shape of the holes. The transmissivity of the $(1,0)$ maximum is increased by almost an order of magnitude and redshifted by roughly $170 \mathrm{~nm}$ when the shape of the holes is changed from circular to rectangular $\left(75 \times 225 \mathrm{~nm}^{2}\right)$. is decreased to $75 \times 225 \mathrm{~nm}^{2}$, the transmissivity of the $(1,0)$ maximum actually increases further to a value of 2.4 , an enhancement with respect to the circular holes of a factor of 9.5. Under the assumption that the maximally transmitted wavelength stays constant, these measured increases are a factor of 5.1 (large rectangles) and of 26.3 (small rectangles) more than the expectations, based on a quadratic relation between hole area and normalized transmission, from conventional transmission theory for (circular) subwavelength holes $[19,20]$. The peak values for the $(1,1)$ and $(2,0)$ peaks also increase when the shape of the holes is altered from circular to rectangular. Clearly, the shape of the holes greatly affects the amount of extraordinary transmission.

Moreover, not only the height of the transmission peaks is affected by the hole shape. The position of the peaks shifts to longer wavelengths when the aspect ratio of the holes is increased. The $(1,0)$ peak shifts by much more than its width as the hole shape in the arrays is changed from circular $\left(\lambda_{(1,0)}=750 \pm 10 \mathrm{~nm}\right)$ via the large rectangles $\left(\lambda_{(1,0)}=840 \pm 10 \mathrm{~nm}\right.$, a wavelength shift of $90 \mathrm{~nm}$, corresponding to an energy difference of $\left.1430 \mathrm{~cm}^{-1}\right)$ to the smallest rectangles $\left(\lambda_{(1,0)}=920 \pm\right.$ $10 \mathrm{~nm}$ ), i.e., a maximum wavelength shift of $170 \pm$ $14 \mathrm{~nm}$ (energy difference of $2460 \mathrm{~cm}^{-1}$ ). All the other peaks also shift to a longer wavelength, albeit to a lesser extent. Current models of extraordinary transmission take into account film thickness and periodicity and use the long wavelength limit so that hole shape can be ignored [13]. As the film thickness and the periodicity were kept constant here, no expectations existed concerning peak shifts and transmission enhancements.

We have therefore performed new calculations that do take the shape of the holes into account. We have used a Fourier modal method (FMM) originally optimized for crossed gratings [21]. In this method the fields in the hole arrays are expanded in a modal basis. The method is closely related to the plane wave method used for photonic crystals [5], but in our case also evanescent modes need to be taken into account. All the modes, including the evanescent ones, are obtained through an eigenvalue problem in the Fourier space of which the eigenvalues give the $z$ component of the $q$ th mode wave vector $\gamma_{q}$ and eigenvectors the field distribution $\phi_{q}(x, y)$. The shape of the holes enter this eigenvalue problem through the Fourier components of the electrical permittivity. In order to deal with highly reflecting materials, the $S$-matrix propagation algorithm is used to deal with the growing evanescent waves in the metal [22]. The convergence of the double Fourier series of the field is improved by using the correct rules for Fourier factoring products that contain discontinuous functions [23,24]. The field components in the hole region are thus written as

$$
E_{\sigma}(x, y, z)=\sum_{q}\left[u_{q} \exp \left(i \gamma_{q} z\right)+d_{q} \exp \left(-i \gamma_{q} z\right)\right] \phi_{q}(x, y)
$$


where $x$ and $y$ are the coordinates along the directions of periodicity of the grating and $z$ is the coordinate along the grating normal, $\sigma=x, y$, and $u_{q}$ and $d_{q}$ are, respectively, the amplitudes of the upward and downward propagating or decaying modal fields (in our actual case all modes are evanescent). The incident and transmitted fields above and below the array are expanded in plane waves. Frequency-dependent permittivities were taken from literature [25].

Figure 3 shows the calculated transmissivities through the arrays used in the experiments for an incident polarization perpendicular to the long axis of the rectangular holes. It is clear that the transmissivity of all the peaks strongly increases when the hole shape is changed from circles to $150 \times 225 \mathrm{~nm}^{2}$ and $75 \times 225 \mathrm{~nm}^{2}$ rectangles. The calculation reproduce the large redshifts of the $(1,0)$ peak as the hole shape is changed from circular to large rectangles (shift $=70 \mathrm{~nm}$ ) and small rectangles $($ shift $=110 \mathrm{~nm})$. The calculated redshifts are slightly smaller than those observed in the experiment. The higher order peaks are less well resolved in the calculations than in the experiment. Overall the theoretical calculations confirm the large effect of hole shape on the extraordinary transmission.

The enhanced transmissivity is found to be strongly polarization dependent. Both the measurements and the calculations show that for the $(1,0)$ peak the transmission for an incident polarization perpendicular to the long axis of the holes is more than 3 orders of magnitude larger than for an incident polarization along the long axis. A similar polarization anisotropy has been observed in the (enhanced) transmission of parallel arrays of slits and wires, both in calculations and experiments (see, e.g., $[26,27])$.

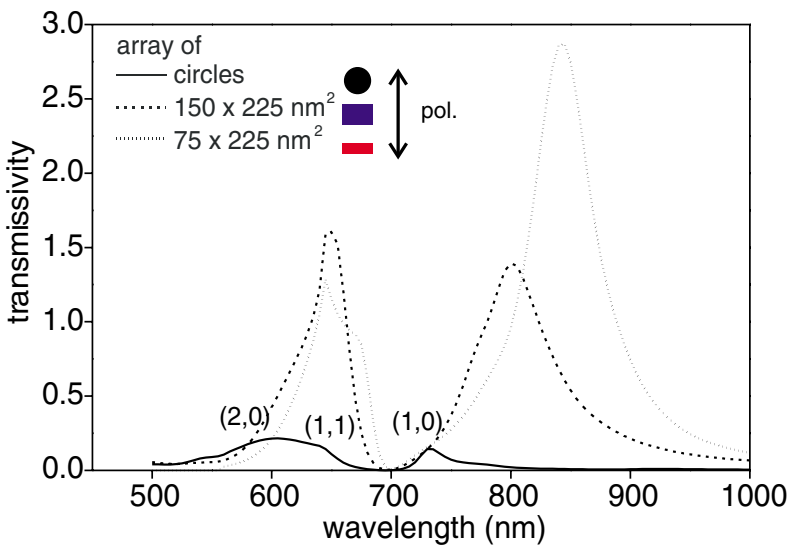

FIG. 3 (color online). Calculated transmissivity spectra for three different subwavelength hole arrays. The spectra are calculated for arrays consisting of circular holes (solid line), $150 \times 225 \mathrm{~nm}^{2}$ rectangles (dotted line), and $75 \times 225 \mathrm{~nm}^{2}$ rectangles (dashed line). The numbers between brackets specify the order of the maxima. The polarization of the incoming light was perpendicular to the long axis of the rectangles.
In order to investigate the role of hole shape on the extraordinary transmission in greater detail, the transmissivity of isolated holes is measured. In order to obtain a signal belonging to a single hole that is significantly larger than the residual transmission of the $200 \mathrm{~nm} \mathrm{Au}$ film, samples are investigated in which a low density of 500 holes are positioned at random positions in an area of $20 \times 20 \mu \mathrm{m}^{2}$. The shape of the holes was the same as those used in the periodic arrays. The relative positions of the holes were equal for the three investigated hole shapes. Figure 4 shows the transmissivity of the random arrays for the different hole shapes used in this investigation. The monotonously decaying transmissivity as a function of wavelength $(\lambda>600 \mathrm{~nm})$ for the random array of circular holes is well characterized with the Bethe-Bouwkamp theory $[19,20]$. The absence of any resonant features in the transmission spectrum of this array shows that intense collective and interference effects in these random arrays can be neglected with respect to the transmission through the individual holes. The total transmission of the random arrays is therefore equal to the sum of the transmission through the isolated holes, so that the transmissivity of an individual hole can be determined. The polarization of the incoming light is perpendicular to the long axis of the rectangular holes. For waveleng ths larger than $600 \mathrm{~nm}$ the rectangular holes have a strong, broad transmissivity that is strongly increased with respect to that of the circular holes.

We attribute the broad increase in transmissivity for the rectangular holes with respect to the circular holes to the existence of shape resonances in the rectangular holes. We have found no evidence for shape resonances in the

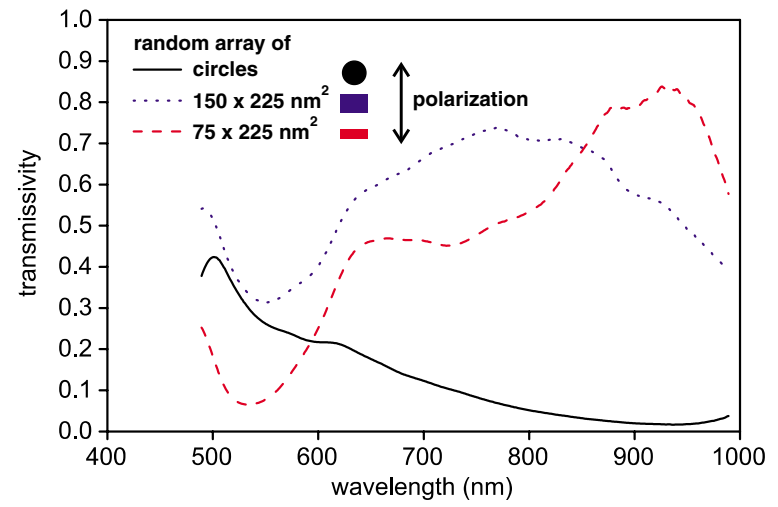

FIG. 4 (color online). Measured transmissivity spectra for patterns of randomly positioned subwavelength holes of different shapes: circular (solid line), $150 \times 225 \mathrm{~nm}^{2}$ rectangles (dotted line), and $75 \times 225 \mathrm{~nm}^{2}$ rectangles (dashed line). The polarization of the incoming light was perpendicular to the long axis of the rectangles. The spectrum of the circular holes is largely featureless and the drop in transmissivity obeys the power law calculated by Bethe [19] and Bouwkamp [20]. For the rectangular holes a broad increase in transmissivity is visible at wavelengths of $600 \mathrm{~nm}$ and larger. The observed increase in transmissivity is maximally 0.8 . 
circular holes. The increase of the transmission and the redshift of the peaks for the arrays can be compared to the spectra of the shape resonances. Under the assumption that the effects from periodicity and shape are separable (in general not the case), at least half of the observed transmissivity increase can be attributed to the shape resonances of the rectangular holes. The strong broad spectral feature for larger wavelengths of the shape resonances suggests that the resonances are also largely responsible for the observed redshift of the $(1,0)$ peak.

The shape resonances observed for the rectangular holes are strongly reminiscent of the localized modes in metal nanoparticles [4]. When the shape of metal particles is changed from spherical to rodlike, this lifts the degeneracy resulting in short- and long-axis modes, where the long-axis modes have an increased oscillator strength and a lower resonance energy [2]. A comparison of the transmissivity of the individual $75 \times 225 \mathrm{~nm}^{2}$ and $150 \times 225 \mathrm{~nm}^{2}$ holes suggests a redshift of the shape resonance for increasing aspect ratio. These redshifted shape resonances are excited with a polarization perpendicular to the long axis, whereas the redshifted longaxis modes for metal nanorods are excited with light polarized parallel to the long axis. This observation seems consistent with complementarity between holes and particles.

In conclusion, we have shown experimentally and theoretically that the shape of the holes on the nanometer scale in ordered metal hole arrays strongly affects the surface-plasmon mediated phenomenon of extraordinary transmission. An increase of almost an order of magnitude in the normalized transmission through optically thick Au films is found when the hole shape is changed from circular (diameter $190 \mathrm{~nm}$ ) to rectangular $75 \times$ $225 \mathrm{~nm}^{2}$ holes, despite the fact that the surface area of the holes decreases. Moreover, we find that the position of the transmission maxima undergoes a large spectral redshift. The experimental findings are reproduced by calculations based on a Fourier modal method for crossed gratings. Measurements on individual rectangular holes show that they exhibit strong shape resonances that are absent for the circular holes. At least half of the magnitude of the effects observed in the ordered arrays can be attributed to these shape resonances. Having the shape of the subwavelength holes as a new parameter in the extraordinary light transmission will extend the potential of these ordered subwavelength structures for sensing, data storage, and light extraction from LEDs [28].

The authors gratefully acknowledge Bas Swinkels who made the first metal hole arrays in our group. L. K. acknowledges John Pendry and Henri Lezec for useful discussions. We thank Adriaan Tip for bringing the Dutch and French collaborators together. The work de- scribed in this Letter is part of and financially supported by the Strategic Research Orientation "Advanced Photonic Structures" of the MESA ${ }^{+}$Research Institute of the University of Twente. This work is part of the research program of the Stichting voor Fundamenteel Onderzoek der Materie (FOM), which is financially supported by the Nederlandse Organisatie voor Wetenschappelijk Onderzoek (NWO).

[1] E. J. Sanchez, L. Novotny, and X. S. Xie, Phys. Rev. Lett. 82, 4014 (1999).

[2] C. Sönnichsen et al., Phys. Rev. Lett. 88, 077402 (2002), and references therein.

[3] J. B. Pendry, A. J. Holden, W. J. Stewart, and I. Youngs, Phys. Rev. Lett. 76, 4773 (1996).

[4] U. Kreibig and M. Vollmer, Optical Properties of Metal Clusters (Springer, Berlin, 1995).

[5] Photonic Crystals and Light Localization in the 21st Century, edited by C. M. Soukoulis, NATO Science Series Vol. 563 (Kluwer, Dordrecht, 2001).

[6] S. C. Kitson, W. L. Barnes, and J. R. Sambles, Phys. Rev. Lett. 77, 2670 (1996).

[7] T.W. Ebbesen et al., Nature (London) 391, 667 (1998).

[8] H. F. Ghaemi et al., Phys. Rev. B 58, 6779 (1998).

[9] D. E. Grupp et al., Appl. Phys. Lett. 77, 1569 (2000).

[10] E. Altewischer, M. P. van Exter, and J. P. Woerdman, Nature (London) 418, 304 (2002).

[11] W. C. Tan, T.W. Preist, and R. J. Sambles, Phys. Rev. B 62, 11134 (2000).

[12] E. Popov, M. Nevière, S. Enoch, and R. Reinisch, Phys. Rev. B 62, 16100 (2000).

[13] L. Martin-Moreno et al., Phys. Rev. Lett. 86, 1114 (2001).

[14] S. A. Darmanyan and A.V. Zayats, Phys. Rev. B 67, 035424 (2003).

[15] O. Toader and S. John, Science 292, 1133 (2001).

[16] O. Toader, M. Berciu, and S. John, Phys. Rev. Lett. 90, 233901 (2003).

[17] R. W. Wood, Phys. Rev. 48, 928 (1935).

[18] H. Raether, Surface Plasmons on Smooth and Rough Surfaces and on Gratings (Springer, Berlin, 1988).

[19] H. A. Bethe, Phys. Rev. 66, 163 (1944).

[20] C. J. Bouwkamp, Philips Res. Rep. 5, 401 (1950).

[21] L. F. Li, J. Opt. Soc. Am. A 14, 2758 (1997).

[22] L. F. Li, J. Opt. Soc. Am. A 13, 1024 (1996).

[23] P. Lalanne and G. M. Morris, J. Opt. Soc. Am. A 13, 779 (1996).

[24] L. Li, J. Opt. Soc. Am. A 13, 1870 (1996).

[25] E. D. Palik, Handbook of Optical Constants in Solids (Academic Press, Boston, 1991), Vol. 1.

[26] P. Gay-Balmaz, C. Maccio, and O. J. F. Martin, Appl. Phys. Lett. 81, 2896 (2002), and references therein.

[27] J. A. Porto, F. J. Garcia-Vidal, and J. B. Pendry, Phys. Rev. Lett. 83, 2845 (1999).

[28] J. Vuckovic, M. Loncar, and A. Scherer, IEEE J. Quantum Electron. 36, 1131 (2000). 